\title{
Analysis of Risk Factors Associated with Cognitive Dysfunction in Patients with Atrial Fibrillation
}

\author{
Jiangfeng Tang1, Xianglin Cheng ${ }^{2 *}$ \\ ${ }^{1}$ Department of Medicine, Yangtze University, Jingzhou, China \\ ${ }^{2}$ Rehabilitation Department of First Affiliated Hospital of Yangtze University, Jingzhou, China \\ Email: ${ }^{*} 45423626 @ q q . c o m$
}

How to cite this paper: Tang, J.F. and Cheng, X.L. (2020) Analysis of Risk Factors Associated with Cognitive Dysfunction in Patients with Atrial Fibrillation. Yangtze Medicine, 4, 319-330.

https://doi.org/10.4236/ym.2020.44030

Received: February 26, 2020

Accepted: September 27, 2020

Published: September 30, 2020

Copyright $\odot 2020$ by author(s) and Scientific Research Publishing Inc. This work is licensed under the Creative Commons Attribution International License (CC BY 4.0).

http://creativecommons.org/licenses/by/4.0/ (c) (i) Open Access

\begin{abstract}
Objective: To discuss the risk factors of cognitive dysfunction in patients with atrial fibrillation. Methods: The 150 cases of patients with atrial fibrillation were analyzed in the first affiliated hospital of Nanchang University who were treated in the cardiovascular department, general medicine department and gerontology department from August 2018 to June 2019. We used Mini-Mental State Examination (MMSE) score to evaluate cognitive function of patients with atrial fibrillation. According to the level of education and MMSE score, patients with atrial fibrillation were divided into three groups: normal cognitive function group, mild cognitive impairment group (MCI) and dementia group. And then the demographic data, the previous use of taking drugs, the results of ultrasonic cardiogram (UCG) and laboratory test were analyzed. Results: 1) The basic situation of research object: a total of 150 patients with atrial fibrillation were enrolled in the study, and the average age of these patients was $65.05 \pm 8.74$ years old, which included 78 males (52\%) and 72 females (48\%). The mean MMSE score was $23.42 \pm 4.65$. According to MMSE score, 86 cases (57.3\%) of cognitive dysfunction occurred in 150 patients with atrial fibrillation, which included 41 cases (27.3\%) of mild cognitive impairment and 45 cases (30\%) of dementia. 2) The comparison of general clinical data: there were significant differences in age, smoking, level of education, left ventricular ejection fraction, left atrial diameter, D-dimer, fibrinogen, homocysteine, platelet and previous use of taking warfarin, dabigatran, $\mathrm{CCB}$, statins among the three groups $(\mathrm{P}<0.05)$. 3) The linear correlational analysis between risk factors of cognitive function and MMSE score in patients with atrial fibrillation: there was a positive correlation between left ventricular ejection fraction and MMSE score, but age, left atrial diameter, homocysteine, low density lipoprotein, platelet, BMI, NT-proBNP, D-dimer
\end{abstract}


were negatively correlated with MMSE score. 4) The risk factors with statistical significance in ANOVA were analyzed by ordinal and multinomial logistic regression, which showed that age $(\mathrm{OR}=1.174,95 \% \mathrm{CI}: 0.091-0.231)$, the level of education (illiteracy OR $=4.162$, 95\% CI: $-0.032-2.955$, primary school OR $=2.751,95 \%$ CI: $-0.172-2.197$, junior high school OR $=3.539$, 95\% CI: $-0.048-2.577$, senior high school and special secondary school OR = 1.332, 95\% CI: -1.080 - 1.655), no CCB (OR = 1.174, 95\% CI: $0.091-0.231$ ), no warfarin $(\mathrm{OR}=13.749,95 \% \mathrm{CI}: 1.480-3.762)$, no dabigatran $(\mathrm{OR}=$ 16.395, 95\% CI: 1.462 - 4.131), D-dimer (OR = 2.745, 95\% CI: $-0.611-2.631)$, fibrinogen ( $\mathrm{OR}=3.228,95 \% \mathrm{CI}: 0.399$ - 1.946) were related to the high occurrence of cognitive dysfunction. Conclusions: 1) Patients with atrial fibrillation had a higher risk of cognitive dysfunction (the incidence of 57.4\%). 2) There was a positive correlation between left ventricular ejection fraction and MMSE score, but age, left atrial diameter, homocysteine, low density lipoprotein, platelet, $\mathrm{BMI}, \mathrm{NT}$-proBNP, and D-dimer were negatively correlated with MMSE score. 3) High level of education, previous use of taking warfarin and dabigatran etexilate were protective factors for cognitive function in patients with atrial fibrillation; but age, previous use of taking CCB, D-dimer and fibrinogen were the risk factors in patients with atrial fibrillation.

\section{Keywords}

Atrial Fibrillation, Cognitive Dysfunction, Dementia, Risk Factors

\section{Introduction}

Atrial fibrillation can cause the symptoms of palpitations and dizziness, and some patients will also bring about cerebral infarction, heart failure and other problems, posing a great threat to human health. We need to improve the strategies for the treatment of atrial fibrillation through more studies on atrial fibrillation and cognitive impairment. Therefore, this study analyzed the risk factors of patients with atrial fibrillation complicated with cognitive dysfunction in order to provide directions for clinical and theoretical research.

\section{Materials and Methods}

\subsection{Research Object}

The 150 cases of patients with atrial fibrillation were analyzed in the first affiliated hospital of Nanchang University who were treated in the cardiovascular department, general medicine department and gerontology department from August 2018 to June 2019. Inclusion criteria: 1) the study was for patients who over 18 years old; 2) atrial fibrillation was diagnosed by the 12-lead electrocardiogram and $24 \mathrm{~h}$ dynamic electrocardiogram by the diagnosis criteria of $\mathrm{P}$ wave disappears, and the spacing off wave is uneven, with different sizes and shapes, frequency up to 300 - 600 times/min; the R-R spacing is absolutely irregular; 
there was no abnormal morphology of QRS wave, and patients with atrial fibrillation complicated with indoor differential conduction presented malformed and wide spacing. Exclusion criteria: 1) the patients less than 18 years old;2) the patients with congenital mental retardation; 3 ) the patients used drugs that affect cognitive function recently; 4) the patients with hepatic encephalopathy, pulmonary encephalopathy, central nervous system infection and other diseases affecting cognitive function; 5) the patients who can't cooperate to complete the cognitive function assessment for various reasons; 6) the patients with schizophrenia, depression and other mental disorders; 7) the patients with serious brain injuries; 8) the patient with repeated hospitalization.

\subsection{Research Method}

\subsubsection{Investigation Method}

Inpatient data of all patients were collected as observational indicators, which included the demographic date, previous use of taking drugs, the results of ultrasonic cardiogram (UCG) and laboratory test. And the cognitive function of patients was evaluated by mini-mental State Examination (MMSE) scale [1]. All patients with atrial fibrillation were divided into three groups according to the level of education and MMSE score, which included normal cognitive function group, MCI group and dementia group.

\subsubsection{Diagnostic Criteria}

The MMSE score of patients in the normal cognitive function group was $\geq 27$, the other patients lower than that was diagnosed with cognitive dysfunction. Different levels of education affect the degree of cognitive function. The MMSE score of patients in dementia group was $\leq 17$ for illiterate, $\leq 20$ for primary school, $\leq 22$ for junior high school, high school and technical secondary school, $\leq 23$ for college and above. And the MMSE score of patients in MCI group was higher than that but lower than normal cognitive function group.

\subsubsection{Quality Control}

The MMSE scale of patients was evaluated by the members of the research group. All pre-evaluation inspectors have received uniform professional training. During the evaluation, it was necessary to explain the purpose of the evaluation to the research object. And we also created a quiet, comfortable, light-appropriate, well-ventilated environment for the patients. More importantly, we should conduct the evaluation on the research object with a neutral and objective attitude. All data entry are double-checked and double entered to ensure the data is accurate.

\subsubsection{Statistical Method}

Data input and management were performed using Excel software, and data analysis was performed using SPSS 21.0 statistical software. The measurement date was represented by $\mathrm{X} \pm \mathrm{S}$. Multiple sets of measurement date were analyzed by one-way ANOVA or Welch's ANOVA. And the enumeration data were ex- 
pressed as count and percentage. The enumeration data were analyzed by chi-square test. We used the Spearman rank correlation for two continuous variables. Otherwise, the Pearson rank correlation was used. The risk factors with statistical significance in ANOVA were analyzed by ordinal and multinomial logistic regression. We adopted a significance level of $\mathrm{P}<0.05$ in all analyses.

\section{Results}

\subsection{The Basic Situation of Research Object}

A total of 150 patients with atrial fibrillation were enrolled in this study, and the average age of these patients was $65.05 \pm 8.74$ years old. We included 78 males (52\%) and 72 females (48\%), and the mean of MMSE score was $23.42 \pm 4.65$. According to MMSE score, 86 cases (57.3\%) of cognitive dysfunction occurred in 150 patients with atrial fibrillation, which included 41 cases $(27.3 \%)$ of mild cognitive impairment and 45 cases $(30 \%)$ of dementia.

\subsection{The Comparison of Demographic Data of Patients with Atrial Fibrillation in Three Groups}

The three groups were compared in age, marital status, gender, BMI, daily smoking, daily drinking and the level of education, among which there were significant differences in age, daily smoking and the level of education $(\mathrm{P}<0.05)$, while significant differences were not found in BMI, marital status, gender and daily drinking $(\mathrm{P}>0.05)$. As shown in Table 1.

Table 1. The comparison of demographic data in three groups (BMI: body mass index; MCI: mild cognitive impairment).

\begin{tabular}{ccccc}
\hline & $\begin{array}{c}\text { Normal Cognitive function } \\
\mathrm{n}=64\end{array}$ & $\begin{array}{c}\text { MCI } \\
\mathrm{n}=41\end{array}$ & $\begin{array}{c}\text { Dementia } \\
\mathrm{n}=45\end{array}$ & $\mathrm{p}$ \\
\hline Age & $59.07 \pm 5.29$ & $67.39 \pm 5.30$ & $71.42 \pm 9.74$ & 0.000 \\
Marital status & & & & 0.061 \\
Married & $60(93.8)$ & $32(78.0)$ & $38(84.4)$ & \\
Unmarried & $4(6.3)$ & $9(22.0)$ & $7(15.6)$ & \\
Gender & & & & 0.968 \\
Men & $33(51.6)$ & $22(53.7)$ & $23(51.1)$ & \\
Women & $31(48.4)$ & $19(46.3)$ & $22(48.9)$ & \\
BMI & $22.64 \pm 1.27$ & $22.42 \pm 1.48$ & $24.06 \pm 1.98$ & 0.070 \\
Daily smoking & $27(42.2)$ & $36(87.8)$ & $37(82.2)$ & 0.000 \\
Daily drinking & $11(17.2)$ & $15(36.6)$ & $15(33.3)$ & 0.052 \\
Level of education & & & & 0.000 \\
Illiteracy & $3(4.7)$ & $6(14.6)$ & $12(26.7)$ & \\
Primary school & $10(15.6)$ & $12(29.3)$ & $12 .(26.7)$ & \\
Junior high school & $5(7.8)$ & $8(19.5)$ & $8(17.8)$ & \\
High school and technical & $8(12.5)$ & $6(14.6)$ & $6(13.3)$ & \\
secondary school & $38(59.4)$ & $9(22.0)$ & $7(15.6)$ & \\
College and above & & & & \\
\hline
\end{tabular}




\subsection{The Comparison of Cardiac Color Doppler Ultrasound and Laboratory Test Results in Three Groups}

In this study, significant differences were found in left ventricular ejection fraction, left atrial diameter, d-dimer, fibrinogen, homocysteine and platelet among the three groups $(\mathrm{P}<0.05)$, as shown in Table 2.

\subsection{The Comparison of Previous Use of Taking Drugs in Three Groups}

In this study, there were significant differences in the previous use of taking warfarin, dabigatran etexilate, $\mathrm{CCB}$ and statins among the three groups $(\mathrm{P}<$ 0.05), as shown in Table 3.

\subsection{The Linear Correlation Analysis between Risk Factors of Cognitive Dysfunction and MMSE Score in Three Groups}

In this study, the results of linear correlation analysis showed that there was a positive correlation between left ventricular ejection fraction and MMSE score.

Table 2. The comparison of cardiac color Doppler ultrasound and laboratory test in three groups.

\begin{tabular}{|c|c|c|c|c|}
\hline & $\begin{array}{l}\text { Normal cognitive function } \\
\qquad \mathrm{n}=64\end{array}$ & $\begin{array}{c}\text { MCI } \\
\mathrm{N}=41\end{array}$ & $\begin{array}{l}\text { Dementia } \\
\mathrm{N}=45\end{array}$ & $\mathrm{p}$ \\
\hline LVEF & $53.70 \pm 9.49$ & $46.26 \pm 7.73$ & $39.05 \pm 4.94$ & 0.000 \\
\hline LAD & $36.18 \pm 3.11$ & $36.17 \pm 4.06$ & $40.01 \pm 4.8$ & 0.000 \\
\hline NT-proBNP & $565.15 \pm 484.18$ & $756.89 \pm 616.80$ & $759.63 \pm 462.87$ & 0.081 \\
\hline D-dimer & $0.36 \pm 0.15$ & $0.41 \pm 0.36$ & $0.57 \pm 0.61$ & 0.027 \\
\hline FIB & $2.40 \pm 0.43$ & $2.19 \pm 0.48$ & $2.79 \pm 0.96 \mathrm{eu}$ & 0.001 \\
\hline $\mathrm{PT}$ & $10.87 \pm 0.82$ & $10.84 \pm 0.96$ & $10.82 \pm 1.15$ & 0.954 \\
\hline CHOL & $4.05 \pm 1.33$ & $4.19 \pm 1.28$ & $4.51 \pm 0.98$ & 0.159 \\
\hline TG & $1.54 \pm 0.77$ & $1.30 \pm 0.72$ & $1.39 \pm 0.75$ & 0.252 \\
\hline HDL & $2.15 \pm 0.50$ & $2.03 \pm 0.44$ & $2.14 \pm 0.45$ & 0.403 \\
\hline LDL & $3.33 \pm 1.34$ & $3.93 \pm 1.48$ & $3.84 \pm 1.42$ & 0.059 \\
\hline ALT & $29.53 \pm 7.93$ & $28.70 \pm 10.51$ & $29.53 \pm 11.04$ & 0.906 \\
\hline AST & $32.17 \pm 7.53$ & $30.78 \pm 9.40$ & $31.00 \pm 9.13$ & 0.650 \\
\hline TBIL & $18.19 \pm 4.29$ & $18.36 \pm 4.81$ & $17.47 \pm 3.29$ & 0.494 \\
\hline Alb & $44.80 \pm 4.17$ & $44.17 \pm 4.43$ & $42.90 \pm 5.64$ & 0.168 \\
\hline Hcy & $8.40 \pm 4.73$ & $16.88 \pm 6.95$ & $19.40 \pm 6.82$ & 0.000 \\
\hline $\mathrm{SCr}$ & $79.96 \pm 14.76$ & $77.67 \pm 13.86$ & $83.17 \pm 14.84$ & 0.213 \\
\hline BUN & $7.00 \pm 0.25$ & $7.01 \pm 0.29$ & $6.95 \pm 0.25$ & 0.606 \\
\hline Plt & $156.26 \pm 36.06$ & $211.97 \pm 22.40$ & $243.40 \pm 41.31$ & 0.000 \\
\hline
\end{tabular}

(LVEF: left ventricular ejection Fractions; LAD: left atrial diameter; NT-proBNP: N-terminal pro-brain natriuretic peptide; FIB: fibrinogen; PT: prothrombin time; CHOL: cholesterol; TG: triglyceride; HDL: high density lipoprotein; LDL: low density lipoprotein; ALT: alanine aminotransferase; AST: aspartate aminotransferase; TBIL: total bilirubin; Alb: albumin; Hcy: hemocyanin; SCr: serum creatinine; BUN: blood urea nitrogen; Plt: platelet; MCI: mild cognitive impairment). 
Table 3. The comparison of previous use of taking drugs in three groups.

\begin{tabular}{ccccc}
\hline & $\begin{array}{c}\text { Normal cognitive function } \\
\mathrm{n}=64\end{array}$ & $\begin{array}{c}\mathrm{MCI} \\
\mathrm{N}=41\end{array}$ & $\begin{array}{c}\text { Dementia } \\
\mathrm{N}=45\end{array}$ & $\mathrm{p}$ \\
\hline Previous use of medication & & & & \\
Aspirin & $17(26.6)$ & $14(34.1)$ & $22(48.9)$ & 0.055 \\
Warfarin & $16(25)$ & $16(39)$ & $7(15.6)$ & 0.045 \\
Dabigatran etexilate & $22(34.4)$ & $5(12.2)$ & $3(6.7)$ & 0.001 \\
Digoxin & $8(12.5)$ & $7(17.1)$ & $10(22.2)$ & 0.406 \\
Beta-blockers & $10(15.6)$ & $12(29.3)$ & $9(20.0)$ & 0.240 \\
Amiodarone & $1(1.6)$ & $3(7.3)$ & $3(6.7)$ & 0.251 \\
ACEI/ARB & $14(21.9)$ & $13(31.7)$ & $12(26.7)$ & 0.530 \\
CCB & $6(9.4)$ & $17(41.5)$ & $15(33.3)$ & 0.000 \\
Statins & $11(17.2)$ & $23(56.1)$ & $13(28.9)$ & 0.000 \\
\hline
\end{tabular}

(ACEI/ARB: angiotensin converting enzyme inhibitors/angiotensin receptor blocker; CCB: calcium-channel blocker; MCI: mild cognitive impairment).

but age, left atrial diameter, homocysteine, low density lipoprotein, platelet, BMI, NT-proBNP, and D-dimer were negatively correlated with MMSE score, as shown in Table 4.

\subsection{The Results of Risk Factors with Statistical Significance in ANOVA Analyzed by Ordinal and Multinomial Logistic Regression}

The results showed that age ( $\mathrm{OR}=1.174,95 \% \mathrm{CI}: 0.091-0.231$ ), level of education (illiteracy OR $=4.162,95 \% \mathrm{CI}:-0.032-2.955$, primary school OR $=2.751$, $95 \%$ CI: $-0.172-2.197$, junior high school OR $=3.539,95 \%$ CI: $-0.048-2.577$, senior high school and special secondary school OR $=1.332,95 \%$ CI: -1.080 1.655), no CCB (OR $=1.174,95 \%$ CI: $0.091-0.231)$, no warfarin $(\mathrm{OR}=13.749$, 95\% CI: 1.480 - 3.762), no dabigatran ( $\mathrm{OR}=16.395,95 \%$ CI: $1.462-4.131)$, D-dimer ( $\mathrm{OR}=2.745,95 \% \mathrm{CI}:-0.611-2.631)$, fibrinogen $(\mathrm{OR}=3.228,95 \% \mathrm{CI}$ : 0.399 - 1.946) were related to the high occurrence of cognitive dysfunction, as shown in Table 5.

\section{Discussion}

Cognitive dysfunction is a kind of disease that influenced by many factors, and the pathogenesis of cognitive dysfunction is very complex. People found that atrial fibrillation may be associated with cognitive dysfunction. Therefore, this study intended to analyze the clinical data of patients with atrial fibrillation, explore the risk factors and protective factors of cognitive dysfunction, and improve the quality of life in patients with atrial fibrillation.

The results of this study found that age was an independent risk factor for cognitive impairment in patients with atrial fibrillation, and with the age increasing, MMSE score showed a decreasing trend. Not only that, in a study by 
Table 4. The linear correlation analysis between risk factors of cognitive dysfunction and MMSE score.

\begin{tabular}{ccc}
\hline Risk factors & $\mathrm{r}$ & $\mathrm{p}$ \\
\hline Age & -0.525 & 0.000 \\
LAD & -0.371 & 0.000 \\
Hcy & -0.530 & 0.000 \\
LDL & -0.237 & 0.004 \\
Plt & -0.669 & 0.000 \\
BMI & -0.215 & 0.008 \\
LVEF & 0.628 & 0.000 \\
NT-proBNP & -0.350 & 0.000 \\
D-dimer & -0.228 & 0.005
\end{tabular}

(LAD: left atrial diameter; Hcy: hemocyanin; LDL: low density lipoprotein; Plt: platelet; BMI: body mass index; LVEF: left ventricular ejection fractions; NT-proBNP: N-terminal pro brain natriuretic peptide).

Table 5. The results of risk factors analyzed by ordinal and multinomial logistic regression.

\begin{tabular}{|c|c|c|c|c|c|c|}
\hline Risk factors & $\begin{array}{l}\text { Regression } \\
\text { coefficient }\end{array}$ & $\begin{array}{l}\text { Standard } \\
\text { error }\end{array}$ & Wald $\chi^{2}$ & $\mathrm{P}$ & OR & $95 \%$ CI \\
\hline Age & 0.161 & 0.036 & 20.105 & 0.000 & 1.174 & $0.091-0.231$ \\
\hline No Smoking & -0.608 & 0.572 & 1.131 & 0.287 & 0.544 & $-1.729-0.512$ \\
\hline $\begin{array}{l}\text { Smoking (reference group) } \\
\text { level of education }\end{array}$ & - & - & - & - & 1 & - \\
\hline Illiteracy & 1.426 & 0.762 & 3.680 & 0.055 & 4.162 & $-0.032-2.955$ \\
\hline Primary school & 1.012 & 0.604 & 2.806 & 0.094 & 2.751 & $-0.172-2.197$ \\
\hline Junior high school & 1.264 & 0.670 & 3.563 & 0.059 & 3.539 & $-0.048-2.577$ \\
\hline $\begin{array}{l}\text { High school and technical } \\
\text { secondary school }\end{array}$ & 0.287 & 0.698 & 0.169 & 0.681 & 1.332 & $-1.080-1.655$ \\
\hline $\begin{array}{l}\text { College and above } \\
\text { (reference group) }\end{array}$ & - & - & - & - & 1 & - \\
\hline No Warfarin & 2.621 & 0.582 & 20.263 & 0.000 & 13.749 & $1.480-3.762$ \\
\hline Warfarin (reference group) & - & - & - & - & 1 & - \\
\hline No dabigatran etexilate & 2.797 & 0.681 & 16.864 & 0.000 & 16.395 & $1.462-4.131$ \\
\hline $\begin{array}{l}\text { Dabigatran etexilate } \\
\text { (reference Group) }\end{array}$ & - & - & - & - & 1 & - \\
\hline No CCB & -0.751 & 0.535 & 1.971 & 0.160 & 0.471 & $-1.800-0.297$ \\
\hline СCB (reference group) & - & - & - & - & 1 & - \\
\hline No Statins & -0.074 & 0.474 & 0.024 & 0.876 & 0.982 & $-1.003-0.855$ \\
\hline Statins (reference group) & - & - & - & - & 1 & - \\
\hline LVEF & -0.019 & 0.041 & 0.217 & 0.641 & 0.981 & $-0.099-0.061$ \\
\hline D-dimer & 1.010 & 0.827 & 1.492 & 0.222 & 2.745 & $-0.611-2.631$ \\
\hline LAD & 0.034 & 0.074 & 0.206 & 0.650 & 1.034 & $-0.112-0.179$ \\
\hline Hcy & 0.138 & 0.040 & 11.734 & 0.001 & 1.148 & $0.059-0.216$ \\
\hline Plt & 0.042 & 0.008 & 26.126 & 0.000 & 1.042 & $0.026-0.058$ \\
\hline FIB & 1.172 & 0.395 & 8.819 & 0.003 & 3.228 & $0.399-1.946$ \\
\hline
\end{tabular}

(CCB: calcium-channel blocker; LVEF: left ventricular ejection fractions; LAD: left atrial diameter; Hcy: hemocyanin; Plt: platelet; FIB: fibrinogen; OR: odds ratio). 
Mathuranath, it was pointed out that age was an independent risk factor for cognitive impairment, this may be related to the fact that elderly patients are prone to hypertension, diabetes, atrial fibrillation and other diseases [2]. At present, most studies support that the harmonious and stable marriage has a protective effect on cognitive function, and Widowhood can reduce the patient's interest of the world, which may increase the risk of cognitive impairment [3] [4]. However, this study did not find an exact relationship between marital status and cognitive dysfunction in patients with atrial fibrillation.

This study also found that daily drinking could not increase the risk of cognitive impairment in patients with atrial fibrillation, which may be related to the fact that these patients were daily drinkers but rarely had an excessive state of drinking. In addition, we found significant difference in daily smoking among the three groups, but it did not become an independent risk factor. Previously, some scholars believed that smoking would not aggravate the impairment of cognitive function and had a protective effect on cognitive function [5]. The protective effect of smoking on cognitive function may be related to the fact that nicotine enhances the antioxidant activity of neurons [6]. In recent years, many studies have also found that smoking may aggravate cognitive impairment. A prospective study showed that smokers had a 1.59-fold higher risk of Alzheimer's disease than non-smokers (95\% CI: 1.15 - 2.20) [7], and the pathogenesis of it may be related to the fact that smoking fume can cause vascular endothelium damage, which aggravates the impairment of cognitive function [8]. The level of education is associated with vocabulary expression, visuospatial structure, the ability of memory and other areas of cognitive function, so it may have significant influence on some neuropsychological tests [9]. The result of this study indicated that high level of education was a protective factor for cognitive functions in patients with atrial fibrillation. The process of education may increase the synaptic connections of neurons, which enable nervous system to tolerate the changes of structure and function [9]. In addition, people with higher level of education are more likely to engage in activities that stimulated cognitive function, and thus people with high level of education have lower rates of cognitive dysfunction compared with low level of education [10]. So moderate mental exercise may help reduce the risk of cognitive impairment in patients with atrial fibrillation. In this study, left atrial diameter was not found to be a risk factor for cognitive dysfunction in patients with atrial fibrillation. As is known to all, left atrial diameter is closely related to many cardiovascular diseases, but it is not clear that whether the increase of left atrial diameter will aggravate the cognitive impairment in patients with atrial fibrillation. Now, in a study by Carlson, it was pointed out that the increase in diameter of left atrium can lead to the formation of thrombosis, and increase the risk of thromboembolic events that affected cognitive function [10]. We also found that there was significant difference in left ventricular ejection fraction among the three groups. The linear correlation analysis also showed a positive correlation between left ventricular ejection fraction and MMSE score, but it was not to the extent of an independent risk factor. 
In a study by Woo, it was pointed out that the structural integrity of synapses in heart failure patients was lower than that in healthy people, and the midbrain circuits were also have changed in the patients [11]. In addition, brain atrophy and demyelination are also common in patients with heart failure, these structural changes are important factors that affected cognitive function and it may contribute to the diagnosis and treatment in patients with cognitive impairment [12] [13].

High blood coagulation state is an important pathogenesis of cerebral vascular embolism caused by atrial fibrillation [14]. Both fibrinogen and D-dimer are important factors involved in the blood coagulation process, and the results of this study indicated that both were risk factors for cognitive dysfunction in patients with atrial fibrillation. Activated platelets can provide phospholipid surfaces for the activation of coagulation factors, and can also release coagulation factors such as fibrinogen. We found that there was a significant difference in platelet among the three groups, the linear Correlation Analysis showed a negative correlation between platelets and MMSE score, and the ordinal and multinomial logistic regression showed that platelet was not a risk factor for cognitive dysfunction in patients with atrial fibrillation. The cognitive impairment caused by high blood coagulation state should be worthy of being attended, which may be an important part of the prevention of cognitive dysfunction in patients with atrial fibrillation. Previously, some scholars found that homocysteine may increase the risk of cardiovascular and cerebrovascular diseases [14]. However, this study not found an exact relationship between homocysteine and cognitive impairment in patients with atrial fibrillation. Because of the high incidence of homocysteine in China, the correlation between homocysteine and cognitive function has become a hot topic in recent years.

Patients with atrial fibrillation are often accompanied by excessive inflammatory response and endothelial cell injury [15], but statins can reduce the level of inflammatory mediators. Therefore, some scholars speculated that statins could have a protective effect on the cognitive function of patients with atrial fibrillation. In a study by Indira Tendolkar, it was pointed out that previous use of taking lipid-regulating drugs in patients with atrial fibrillation could slow the cognitive impairment [16]. In a study by Chao, it was pointed out that, the incidence of non-vascular dementia was lower in patients with atrial fibrillation who received statins, compared with patients who were not received statins [17]. But in this study, we do not find any protective effect of statins on cognitive function in patients with atrial fibrillation, which may be related to many factors such as insufficient time of using statins and different types of statins could have different effects on the cognitive function in patients with atrial fibrillation.

Anticoagulant therapy has long been considered as the cornerstone of atrial fibrillation treatment. In a study by Mavaddat, it was not found that warfarin had protective effect on cognitive function [18]. However, another study involved 2605 patients showed that the time of INR in the treatment range (TTR) 
was significantly correlated with cognitive impairment in patients with atrial fibrillation. It means that the patients with TTR $<25 \%$ could have a higher risk of dementia than with TTR $>70 \%$ [19]. The effect of warfarin on cognitive function may be influenced by many factors and closely related to the time of INR in the treatment range (TTR). However, the new oral anticoagulant has certain advantages in safety and efficacy, and the compliance of that is higher than warfarin. A meta-analysis published by Wenke Cheng showed that previous use of taking dabigatran etexilate had a better protective effect on cognitive function in patients with atrial fibrillation than warfarin. And for patients with atrial fibrillation whose INR was controlled at $2-3$, the increase of TTR was inversely proportional to the incidence of cognitive dysfunction [20]. In this study, the ordinal and multinomial logistic regression showed that dabigatran etexilate and warfarin were protective factors for cognitive dysfunction in patients with atrial fibrillation. Previously, some studies have shown that antiplatelet drugs could be used to prevent stroke in patients with atrial fibrillation, but the results of this study not found that aspirin had a protective effect on cognitive function in patients with atrial fibrillation. So the effect of aspirin on cognitive function in patients with atrial fibrillation needs to be further confirmed by more large-scale multicentre studies.

\section{Conclusion}

1) Patients with atrial fibrillation had a higher risk of cognitive dysfunction (the incidence of 57.4\%). 2) There was a positive correlation between left ventricular ejection fraction and MMSE score. But age, left atrial diameter, homocysteine, low density lipoprotein, platelet, BMI, NT-proBNP, and D-dimer were negatively correlated with MMSE score. 3) The results showed that high level of education, previous use of taking warfarin and dabigatran etexilate were protective factors for cognitive function in patients with atrial fibrillation; age, previous use of taking CCB, D-dimer and fibrinogen were risk factors for cognitive function in patients with atrial fibrillation.

\section{Conflicts of Interest}

The authors declare no conflicts of interest regarding the publication of this paper.

\section{The Claim of Ethical Clearance}

This thesis is an original research, does not contain any forgery, cheating and plagiarism, and does not participate in any violation of academic ethics. This thesis does not involve state secrets, and does not involve any infringement related to intellectual property rights. We guarantee that the paper has not been published in any other journal or media in any other language. And we also guarantee that the paper will not submit to other publications before receiving rejection notice from the editorial department. 


\section{References}

[1] Fernandes, R.C., Da, S.K., Bonan, C., et al. (2009) Cognitive Function in Menopausal Women Evaluated with the Mini-Mental State Examination and Word-List Memory Test. Cadernos De Saúde Pública, 25, 1883-1893. https://doi.org/10.1590/S0102-311X2009000900003

[2] Mathuranath, P.S., George, A., Ranjith, N., et al. (2012) Incidence of Alzheimer's Disease in India: A 10 Years Follow-Up Study. Neurology India, 60, 625-630. https://doi.org/10.4103/0028-3886.105198

[3] Van den Berg, G.J., Lindeboom, M. and Portrait, F. (2011) Conjugal Bereavement Effects on Health and Mortality at Advanced Ages. Journal of Health Economics, 30, 774-794. https://doi.org/10.1016/j.jhealeco.2011.05.011

[4] Ha, J.H. and Ingersoll-Dayton, B. (2011) Moderators in the Relationship Between Social Contact and Psychological Distress among Widowed Adults. Aging \& Mental Health, 15, 354-363. https://doi.org/10.1080/13607863.2010.519325

[5] Mayeux, R. (2004) S1-03-05 Epidemiology of Alzheimer's Disease. Neurobiology of Aging, 25, S6. https://doi.org/10.1016/S0197-4580(04)80020-4

[6] Liu, Y., Zeng, X., Hui, Y., et al. (2015) Activation of Alpha7 Nicotinic Acetylcholine Receptors Protects Astrocytes against Oxidative Stress-Induced Apoptosis: Implications for Parkinson's Disease. Neuropharmacology, 91, 87-96. https://doi.org/10.1016/j.neuropharm.2014.11.028

[7] Peters, R., Poulter, R., Warner, J., et al. (2008) Smoking, Dementia and Cognitive Decline in the Elderly, a Systematic Review. BMC Geriatrics, 8, 36.

https://doi.org/10.1186/1471-2318-8-36

[8] Jenner, P. (2003) Oxidative Stress in Parkinson's Disease. Annals of Neurology, 53, S26-S38. https://doi.org/10.1002/ana.10483

[9] Ardila, A. and Moreno, S. (2001) Neuropsychological Test Performance in Aruaco Indians: An Exploratory Study. Journal of the International Neuropsychological Society, 7, 510-515. https://doi.org/10.1017/S1355617701004076

[10] Carlson, M.C., Helms, M.J., Steffens, D.C., et al. (2008) Midlife Activity Predicts Risk of Dementia in Older Male Twin Pairs. Alzheimer's \& Dementia, 4, 324-331. https://doi.org/10.1016/j.jalz.2008.07.002

[11] Woo, M.A., Palomares, J.A., Macey, P.M., et al. (2015) Global and Regional Brain Mean Diffusivity Changes in Patients with Heart Failure. Journal of Neuroscience Research, 93, 678-685. https://doi.org/10.1002/jnr.23525

[12] Vogels, R.L., van der Flier, W.M., van Harten, B., et al. (2007) Brain Magnetic Resonance Imaging Abnormalities in Patients with Heart Failure. European Journal of Heart Failure, 9, 1003-1009. https://doi.org/10.1016/j.ejheart.2007.07.006

[13] Zamrini, E., De Santi, S. and Tolar, M. (2004) Imaging Is Superior to Cognitive Testing for Early Diagnosis of Alzheimer's Disease. Neurobiology of Aging, 25, 685-691. https://doi.org/10.1016/j.neurobiolaging.2004.02.009

[14] Barber, M., Tait, R.C., Scott, J., et al. (2004) Dementia in Subjects with Atrial Fibrillation: Hemostatic Function and the Role of Anticoagulation. Journal of Thrombosis and Haemostasis, 2, 1873-1878. https://doi.org/10.1111/j.1538-7836.2004.00993.x

[15] Hui, D.S., Morley, J.E., Mikolajczak, P.C. and Lee, R. (2015) Atrial Fibrillation: A Major Risk Factor for Cognitive Decline. American Heart Journal, 169, 448-456. https://doi.org/10.1016/j.ahj.2014.12.015

[16] Tendolkar, I., Enajat, M., Zwiers, M.P., et al. (2012) One-Year Cholesterol Lowering 
Treatment Reduces Medial Temporal Lobe Atrophy and Memory Decline in Stroke-Free Elderly with Atrial Fibrillation: Evidence From a Parallel Group Randomized Trial. International Journal of Geriatric Psychiatry, 27, 49-58.

https://doi.org/10.1002/gps.2688

[17] Chao, T.F., Liu, C.J., Chen, S.J., et al. (2015) Statins and the Risk of Dementia in Patients with Atrial Fibrillation: A Nationwide Population-Based Cohort Study. International Journal of Cardiology, 196, 91-97.

https://doi.org/10.1016/j.ijcard.2015.05.159

[18] Mavaddat, N., Roalfe, A., Fletcher, K., et al. (2014) Warfarin versus Aspirin for Preven tion of Cognitive Decline in Atrial Fibrillation: Randomized Controlled Trial (Birmingham Atrial Fibrillation Treatment of the Aged Study). Stroke, 45, 1381-1386. https://doi.org/10.1161/STROKEAHA.113.004009

[19] Jacobs, V., Woller, S.C., Stevens, S., et al. (2014) Time Outside of Therapeutic Range in Atrial Fibrillation Patients Is Associated with Long-Term Risk of Dementia. Heart Rhythm, 11, 2206-2213. https://doi.org/10.1016/j.hrthm.2014.08.013

[20] Cheng, W., Liu, W., Li, B. and Li, D. (2018) Relationship of Anticoagulant Therapy with Cognitive Impairment among Patients with Atrial Fibrillation: A $\mathrm{Me}$ ta-Analysis and Systematic Review. Journal of Cardiovascular Pharmacology, 71, 380-387. https://doi.org/10.1097/FJC.0000000000000575 